\title{
Evolution of the Immune Status in Children Infected with HIVIAIDS on Antiretroviral Treatment in Lubumbashi, Democratic Republic of Congo
}

\author{
Mbayo Lukasu ${ }^{1}$, Kazadi Mwadianvita ${ }^{2}$, Kalumba Kambote ${ }^{1}$, Kasamba Ilunga ${ }^{2}$, Kabongo Katabwa ${ }^{1}$, Ntambue \\ Mukengeshayi ${ }^{3}$ and Wembonyama Okitosholo ${ }^{1}$ \\ 1. Center of Excellence in Care of HIV/AIDS, University of Lubumbashi, Lubumbashi 1885, Democratic Republic of Congo \\ 2. Department of Laboratory, University of Lubumbashi, Lubumbashi 1885, Democratic Republic of Congo \\ 3. School of Public Health, University of Lubumbashi, Lubumbashi 1885, Democratic Republic of Congo
}

\begin{abstract}
The objective was to describe the immunological status among children infected with HIV (human immunodeficiency virus) at baseline and their change during the treatment. This longitudinal study conducted at the Center of Excellence in Lubumbashi from 2nd semester, 2011 to the 2nd semester, 2012, examined the records of children who had been receiving antiretroviral therapy for 48 weeks. A count of CD4 + T lymphocytes was performed at the beginning, 24 weeks, and 48 weeks of treatment. The immunological status was defined by the 1996 CDC (centers for disease control) immunological categories. All the children were on zidovudine, lamivudine/nevirapine or Efavirens. At initiation of treatment, severe immunosuppression was found in 56\%, moderative in $28 \%$ and lacked in $16 \%$ of cases. Furthermore, after 48 weeks of treatment, a change in the immunological status was observed in $42 \%$ of cases. In contrast, $58 \%$ of cases kept in their original status. In this cohort, the immune status has not undergone significant change.
\end{abstract}

Key words: HIV infection, antiretroviral therapy, immunological status, Lubumbashi.

\section{Introduction}

Pediatric HIV/AIDS (human immunodeficiency virus/acquired immunodeficiency syndrom) is a public health problem worldwide and in the DRC (Democratic Republic of Congo) in particular. The WHO (World Health Organization) estimated that 3.4 million children live with HIV worldwide, with over $90 \%$ in sub-Saharan Africa [1]. In the DRC, the National Programme for Fighting against HIV/AIDS estimated the number of children under 15 infected with HIV at 110,000 including about 38,950 eligible, only 5,315 benefit from ART (antiretroviral therapy) [2]. In Lubumbashi, the seroprevalence of HIV infection was estimated at $7 \%$ and that of the children at $3.7 \%$ as it is correlated to that of the

Corresponding author: Mbayo Lukasu, junior, clinical officer, research field: HIV clinic. E-mail: mbayoxavier@yahoo.fr; xaviermulamba@gmail.com. pregnant woman [3]. Effective ART leads to a gradual and slower an increase of CD4 $+\mathrm{T}$ lymphocytes in patients whose way of advanced immunosuppression has evolved extended [4]. The objective was to describe the immunological status among children infected with HIV at baseline and their changes during 48 weeks of antiretroviral therapy.

\section{Material and Methods}

\subsection{Framework and Type of Study}

The study was conducted at the CE (Center of Excellence) of the University of Lubumbashi integrated with Jason Sendwe Hospital which is the largest public hospital in Lubumbashi. This structure provides medical consultations and takes care freely in charge of persons living with HIV (PLHIV) without exclusivity age and sex. 
This is a longitudinal study of the records of children receiving ART conducted during the period from 2nd semester, 2011 to 2 nd semester, 2012. That is to say 48 weeks (W48).

\subsection{Study Population}

The study population has consisted of 36 HIV-infected children aged 2-15 years, whose HIV status have been tested and confirmed by strategies and national algorithm [5] and have been put on treatment according to clinical and immunological criteria in application in DRC [2]. The children who had been included in this study are children registered in the 2nd half of 2011, which have started ART at the Center of Excellence, followed for 48 weeks of treatment and made at least one $\mathrm{CD} 4+\mathrm{T}$ lymphocytes after initiation treatment.

\subsection{Data Collection}

The collection was carried out through a grid that incorporated the following parameters to be studied: sociodemographic (age and sex); clinics (diseases and OIs (opportunistic infections) diagnosed at the beginning and during follow-up were classified according to clinical stages I, II, III and IV of the WHO [6]). Biologically, (the typing of CD4 $+\mathrm{T}$ lymphocytes was analyzed using a BD Facs count (Becton Dickinson) at the beginning, 24 weeks and 48 weeks of ART. Immunological status was defined by immunological categories of CDC (centers for disease control) in 1994, and was ranked category 3, 2 and 1, respectively in severe immunosuppression $(<15 \%)$, moderative immunosuppression (15\% to $24 \%$ ), and lack of immunosuppression ( $\geq 25 \%$ ) [7]. All children had received the same triple generic therapy: nevirapine $120 \mathrm{mg} \cdot \mathrm{m}^{-2}$ twice daily; zidovidune 180 $\mathrm{mg} \cdot \mathrm{m}^{-2}$ twice daily; lamividune $4 \mathrm{mg} \cdot \mathrm{m}^{-2}$ twice daily with the possibility of alternative molecules. Any children had benefited from the viral load before and during ART.

\subsection{Statistical Analysis}

The collected data were entered and analyzed using Epi Info 3.5.1 software. The usual descriptive statistics were expressed as mean for quantitative variables and the proportions for categorical variables. The Excel 2010 spreadsheet has helped the authors to make the graphics. Patient characteristics at initiation of antiretroviral treatment were compared using the chi-square test $X^{2}$.

\section{Results}

\subsection{General Characteristics of HIV-Infected Children}

Forty-five HIV-infected children before ART were examined and 36 were selected who responded to the criteria of selection. The average age was $8 \pm 3.5$ years (2-15 years) and the sex ratio was 1 . On the 36 remaining HIV-infected children, follow-up data were immunologically available at initiation, at 24 weeks and at 48 weeks of ART.

\subsection{Evaluation of Clinical and Immunological} Parameters before the ART

The mean of CD4 $+\mathrm{T}$ lymphocytes average were $20 \%, 16 \%$ and $14 \%$, respectively in different categories of immunosuppression 3,2 , and 1 of immunological classification of CDC 1994. The immunological status in HIV-infected children were $56 \%, 28 \%$ and $16 \%$, respectively in the category of immunosuppression 3, 2, and 1 of immunological staging of CDC 1994. The opportunistic infections which were the most observed were thrush (7\%), pneumocystosis (5\%), tuberculosis (3\%), and the protein calorie malnutrition (19\%).

\subsection{Evaluation on ART}

Clinically, a case of tuberculosis was observed on 36 HIV-infected children. Immunologically, on 36 observations of the mean of CD4 + T lymphocytes, it was observed an upward immunological profile of average rates mean of CD4 + T lymphocytes to $20 \%$, $24 \%$, and $26 \%$, respectively, which was an increase of 
$4 \%$ and $6 \%$ compared to baseline in the category 1. In category 2 , it was observed $16 \%, 20 \%$, and $22 \%$, respectively, which was an increase of $4 \%$ and $6 \%$. In category 3 , it was noted a downward immunological profile of average rates mean of CD4 + T lymphocytes to $20 \%, 16 \%$ and $12 \%$, respectively, which was a decrease of $2 \%$ and $8 \%$. There are not statistically significant differences in different immunological categories of CDC 1994 (Fig. 1).

These increases of CD4 + T lymphocytes had led to changes in the immunological status in different immunological status CDC 1994 after 48 weeks of treatment in $42 \%$ of cases while $58 \%$ of cases had kept their original status despite the increase or the decrease (Fig. 2).

\section{Discussion}

In DRC, the management of patients infected with HIV is coordinated by the National Programm for Fighting against Sexually Transmited Infections and HIV/AIDS. Since 2005, under the initiative was to put three million people on antiretroviral by the end of 2005 ( 3 by 5), ART is issued free of charge to patients. The observations made in this study are compared

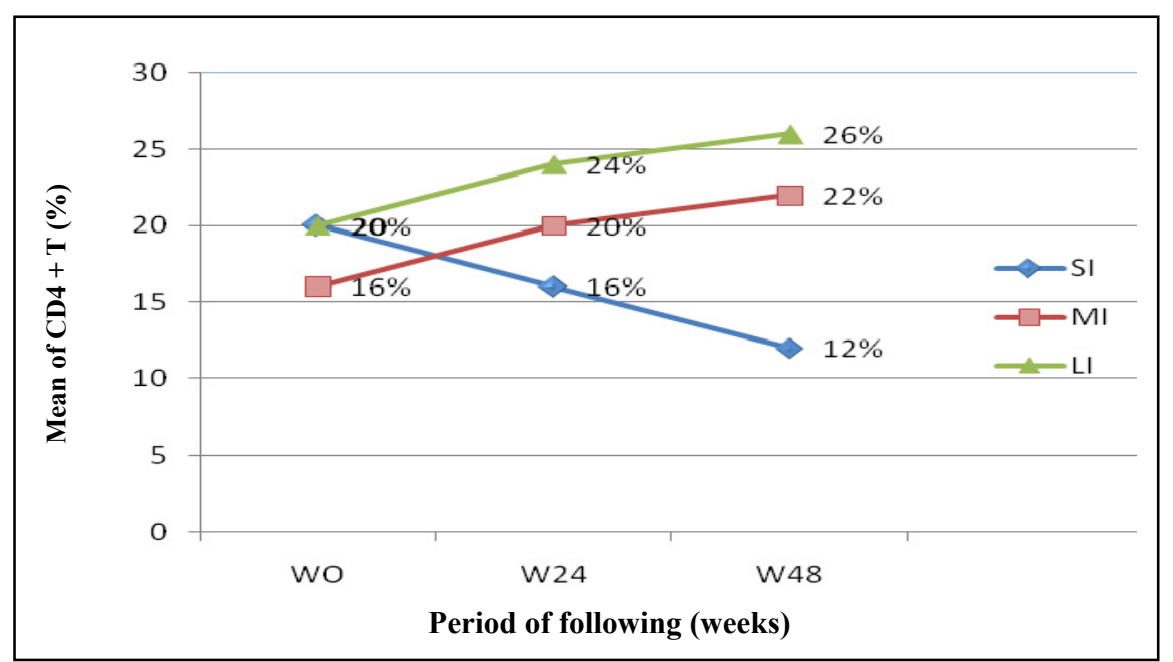

Fig. 1 Evolution of the immunological profile in different categories under antiretroviral treatment during 48 weeks. SI: severe immunosuppression; MI: moderative immunosuppression; LI: lacked immunosuppresion.

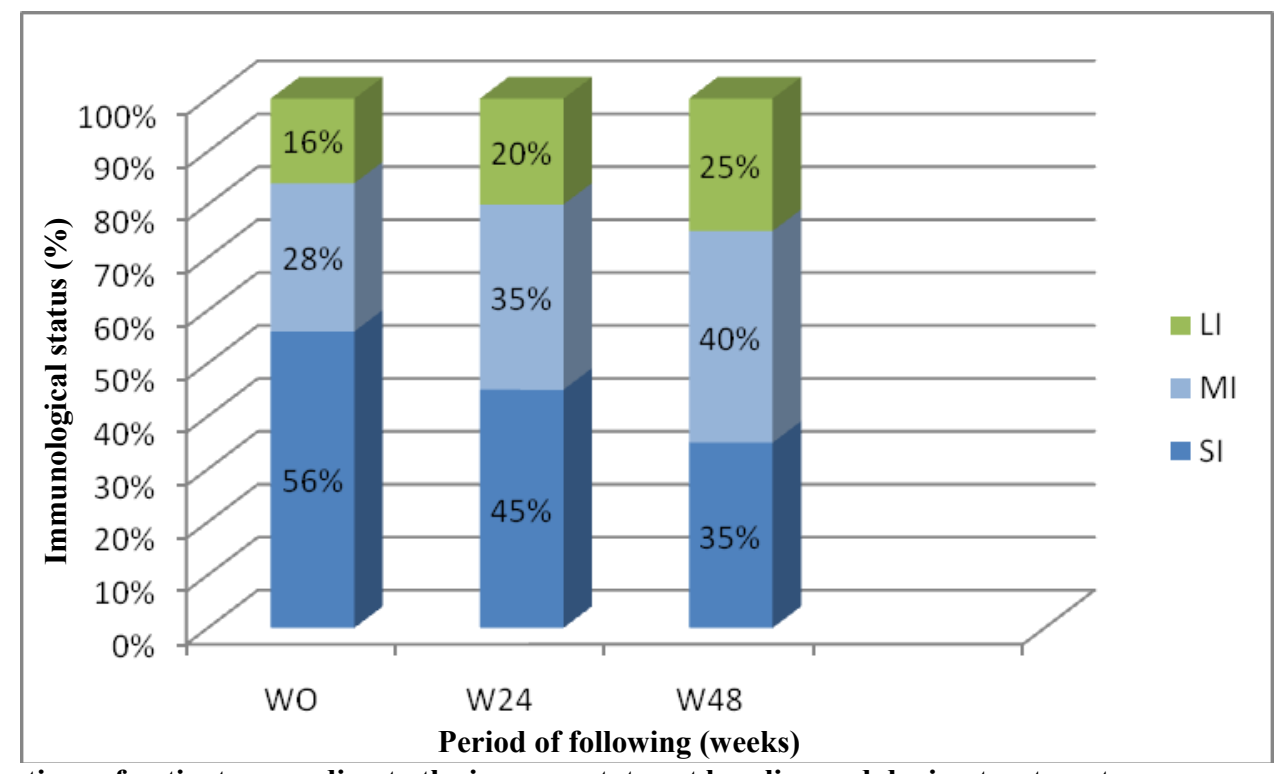

Fig. 2 Distributions of patients according to the immune status at baseline and during treatment.

SI: severe immunosuppresion; MI: moderative immunosuppresion; LI: lacked immunosuppresion. 
with literature data. The average age of the children was $8 \pm 3.5$ years. Other researchers such as Brigita and Seth [8], and Djadou and Agbèrè [9] in Togo had found that the average age is 6 years, 3 months and 7.7 years. Kumarasamy and Solomon [10] in the south of India noted in their series: oral candidiasis is $29 \%$, tuberculosis is $13.4 \%$. In this cohort, it is observed that oral candidiasis is $7 \%$, tuberculosis is $3 \%$, pneumocystosis is $5 \%$ of protein and calorie malnutrition is $19 \%$ at the inclusion. In the course of treatment, 36 children are observed on case of tuberculosis $(3 \%)$. The immunological response allowed recovery of immunological status CDC 1994. But these differences were not significant due to small sample size. Moreover, Song and Shaffiq [11] had noted high virological and immunological discrepancies despite a very significant increase of $\mathrm{CD} 4+\mathrm{T}$ lymphocytes in their series. The immune status has changed in $42 \%$ of cases overall. This suggests that the improvements of $\mathrm{CD} 4+\mathrm{T}$ lymphocytes occurred regardless of their immunological status. In this context, it is important to enjoin Djadou and Agbèrè [9] in Togo who had advocated improving compliance by the social workers and the psychological care of children refusing to take medications to their parents explaining the importance of adherence during home visits. The other $58 \%$ of cases had kept their original status despite the increase or the decrease of CD4 $+\mathrm{T}$ lymphocytes. These results suggest that further investigation with a larger sample would add one more to the study by explaining the relationship between the initial factors for initiation of ART and therapeutic response in midst. The prescribed ART in this study complies with WHO 2009 and consists of zidovudine, lamivudine/nevirapine or efavirens, which are very generic combination prescribe in the country and limited resource such as DRC, because it is lower cost, availability, and proven effectiveness. Limit: the size of this sample and the follow-up period are relatively short and limited the statistical power.
However, a prospective study with a larger sample is needed in Lubumbashi.

\section{Conclusions}

The results reveal a change in the immunological status of CDC 1994 in $42 \%$ of cases in overall induce by an increase of $\mathrm{CD} 4+\mathrm{T}$ lymphocytes under antiretroviral therapy. The authors emphasize the typing of $\mathrm{CD} 4+\mathrm{T}$ lymphocytes in the initiation, monitoring and qualification of immunological status on antiretroviral treatment. In conclusion, the results of this study suggest that further investigation with a larger sample would add one more to the study by explaining the relationship between the initial factors for initiation ART and therapeutic response in Lubumbashi.

\section{Acknowledgments}

The authors express their sincere thanks to the staff of the Centre of Excellence in HIV/AIDS and the Clinical Laboratory of the University of Lubumbashi for their technical support and collaboration.

\section{References}

[1] WHO (World Health Organisation). 2010. Global HIV/AIDS Epidemic. Annual report.

[2] NPF HIV/AIDS (National Program for the Fight against HIV/AIDS). 2010. Guidelines for Management of Opportunistic Infections and Other Diseases Related to in HIV-Infected Children in Democratic Republic of Congo. Kinshasa: NPF HIV/AIDS.

[3] National Program for the Fight against HIV/AIDS. 2008. Sentinel Surveillance of HIV/AIDS in Kinshasa. Health report.

[4] Chirouze, C., and Hoen, B. 2006. "HIV Infection." Review of the Practitioner 56 (9): 941-1012.

[5] NPF HIV/AIDS (National Program for the Fight against HIV/AIDS). 2007. Guide for the Use of Rapid Testing/Diagnosis of HIV Infection. Democratic Republic of Congo: NPF HIV/AIDS.

[6] World Health Organisation. 2006. "Antiretroviral Therapy of HIV Infection in Infants and Children in Resource-Limited Setting towards Universal Access." WHO. Accessed July 4, 2008. http://www.who.int/hiv/pub/guidelines/art/en/. 


\section{Lubumbashi, Democratic Republic of Congo}

[7] Center for Disease Control and Prevention. 1994. "Revised Classification System for Human Immunodeficiency Virus Infection in Children Less Than 13 Years of Age." Center for Disease Control and Prevention. Accessed July 2, 2008. http://www.cdc.gov. mmwr/preview/mmwrhtml/0032890.htm.

[8] Brigita, U. M., and Seth, S. 1998. "A Study of Phase I/II Protease in Inhibitor Ritonavir in Children Infected with Human Immunodeficiency." Pediatrics. 1013 (10): $335-43$.
[9] Djadou, K. E., and Agbèrè, A. D. 2012. "Follow-up HIV-Infected Children Receiving Antiretroviral Therapy in a Rural Area of Togo." Trop Med In Health 22: 283-6.

[10] Kumarasamy, N., and Solomon, S. 2009. "Safety, Tolerability and Effectiveness of Generic in HAART in HIV-Infected Children in South India." J Trop Pediatric 55 (3): 155-9.

[11] Song, R., and Shaffiq, E. 2007. "Efficacy of Highly Active Antiretroviral Therapy in HIV-1 Infected Children in Kenya." Pediatrics 120: 856-61. 